\title{
Leading Neutron Production at ZEUS
}

\author{
W. Schmidke \\ Max Planck Institute for Physics \\ Munich, Germany
}

\begin{abstract}
ZEUS results on leading neutron production in $e p$ scattering are presented. Production in deep inelastic scattering and photoproduction are compared, giving indications of absorption. The data are compared to Monte Carlo, meson exchange and absorption models.
\end{abstract}

\section{Introduction}

Events with a neutron carrying a large fraction of the proton energy have been observed in $e p$ scattering at HERA [1]. The dynamical mechanisms for their production are not completely understood. They may be the result of hadronization of the proton remnant, conserving baryon number in the final state. Exchange of virtual isovector mesons is also expected to contribute, predominantly the exchange of low mass $\pi^{+}$mesons [2]. In this picture the proton fluctuates into a virtual $n-\pi^{+}$state. The virtual $\pi^{+}$scatters with the projectile lepton, leaving the fast forward neutron in the final state. Depending on the virtuality of the exchanged photon, which is a measure of how pointlike the photon is, the neutron may also rescatter with it and migrate to a region outside of the detector acceptance, leading to a depletion of neutrons in some kinematic regions [3].

The ZEUS experiment at HERA had a forward neutron calorimeter (FNC) in the proton beam direction. It measured the fraction of the beam energy carried by the neutron, $x_{L}$, and the transverse momentum transferred to the neutron, $p_{T}$. HERA machine elements along the neutron flight path limited neutron scattering angles to $\theta_{n}<0.75 \mathrm{mrad}$, restricting measurement to the kinematic region $p_{T}^{2}<0.476 x_{L}^{2} \mathrm{GeV}^{-2}$. Here we report results on leading neutron production, in both photoproduction, where the photon virtuality $Q^{2}$ is nearly zero, and in deep inelastic scattering (DIS), where $Q^{2}$ is greater than a few $\mathrm{GeV}^{2}$. Comparisons are made to Monte Carlo models, one of which incorporate the pion exchange mechanism. Comparisons are also made to models of pion exchange incorporating rescattering, and a model including exchanges of additional mesons.

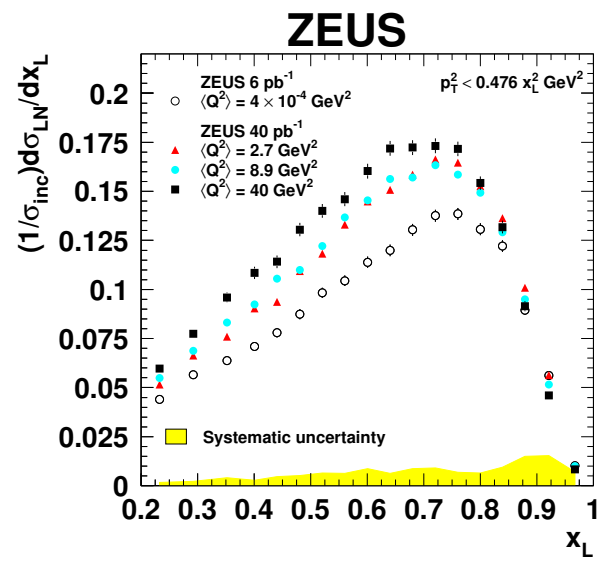

Figure 1: Neutron $x_{L}$ distributions for photoproduction and three $Q^{2}$ bins of DIS. 


\section{Results}

Leading neutron $x_{L}$ distributions for photoproduction and three $Q^{2}$ bins of DIS are shown in Fig. 1. The distributions are normalized by $\sigma_{\text {inc }}$, the inclusive cross section without the neutron requirement. The distributions all rise from lowest $x_{L}$ due to the increase in $p_{T}^{2}$ space, reach a maximum near $x_{L}=0.7$, and fall to zero at the kinematic limit $x_{L}=1$. There is a clear increase of the relative neutron yield with $Q^{2}$. This is consistent with absorption models, where at lower $Q^{2}$ the larger photon size can result in rescattering of the neutron, where the neutron can migrate to a region outside of the detector acceptance and is lost.

For further studies the full $Q^{2}$ range of the DIS sample is taken together. The ratio of the photoproduction $x_{L}$ distribution to that of the full DIS sample is shown in Fig. 2. The depletion of neutrons in the photoproduction sample increases with decreasing $x_{L}$. In pion exchange models the size of the virtual $n-\pi^{+}$ system is smaller at lower $x_{L}$, with increased probability of rescattering. Thus the depletion of neutrons in photoproduction is consistent with pion exchange models including absorption.

The $p_{T}^{2}$ distributions of neutrons in photoproduction and DIS are shown for several $x_{L}$ bins in Fig. 3. They are normalized to unity at $p_{T}^{2}=0$. The lines are fits to exponentials in $p_{T}^{2}$, which give a good description of the data. The photoproduction distributions are steeper in the range $0.6<x_{L}<0.9$, with relatively fewer neutrons at high $p_{T}^{2}$. This is qualitatively consistent with absorption, where rescattering is more likely for the small $n-\pi^{+}$separations corresponding to higher $p_{T}^{2}$.

\section{Comparison to models}

The normalized neutron distributions in DIS were fit to the form

$$
\frac{1}{\sigma_{\text {inc }}} \frac{d \sigma_{\mathrm{LN}}}{d x_{L} d p_{T}^{2}}=a e^{-b p_{T}^{2}} .
$$

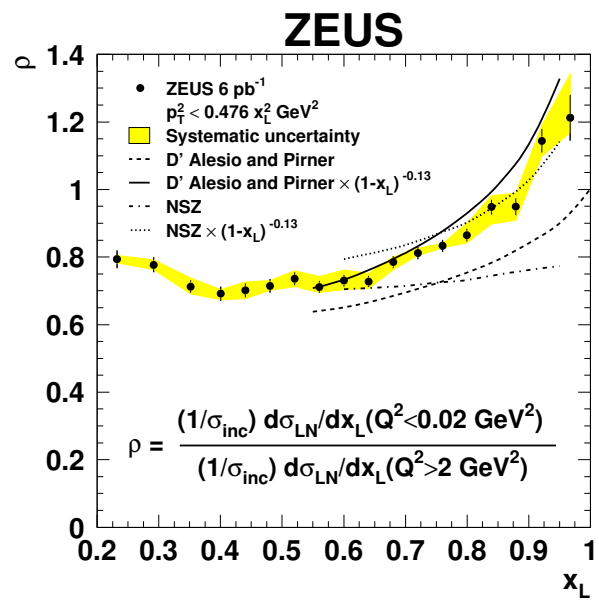

Figure 2: Ratio of neutron $x_{L}$ distributions in photoproduction and DIS.

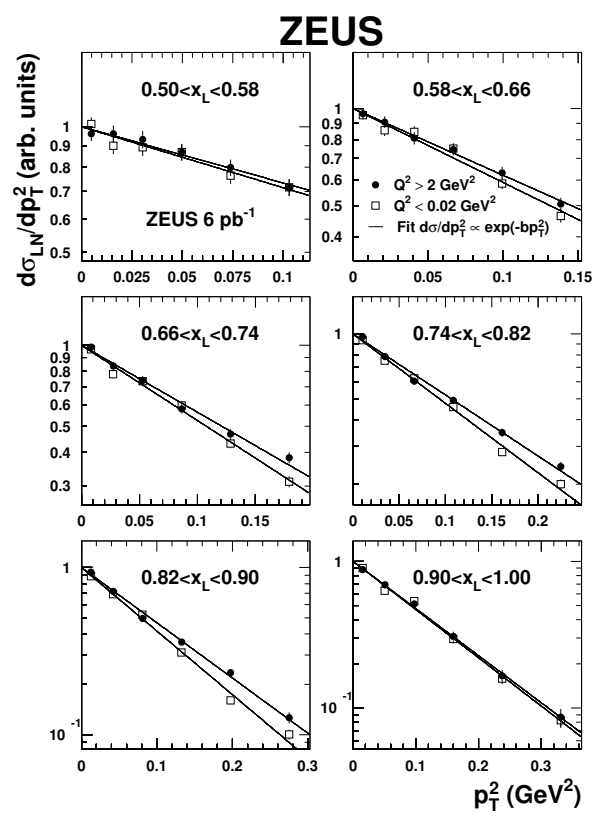

Figure 3: Neutron $p_{T}^{2}$ distributions for photoproduction and DIS, normalized to unity at $p_{T}^{2}=0$. 
Figure 4 shows the $x_{L}$ distributions for $\theta_{n}<0.75 \mathrm{mrad}$ and the intercepts $a$ and slopes $b$ as a function of $x_{L}$. Also on the plots are the expectations of the LEPTO [4] and RAPGAP [5] Monte Carlo models. Both were run in the configuration where neutron were produced from the fragmentation of the proton remnant [6]. LEPTO was also run implementing soft color interactions [?]; RAPGAP was also run including neutron production via pion exchange. Both models with only fragmentation of the proton remnant do not describe the data. They predict too few neutrons, peaked at lower $x_{L}$ than the data. They do not have the broad plateau in intercepts $a$ that the data exhibit for medium values of $x_{L}$, and they predict slopes $b$ smaller than and without the steep $x_{L}$ dependence of the data. LEPTo including soft color interactions gives an $x_{L}$ distribution which better describes the peak in the data and has a slight enhancement of $a$ at medium $x_{L}$ value. RAPGAP with pion exchange gives a good description of the shapes of all distributions, although it predicts more neutrons with larger intercepts and slopes than the data.

The ratio of $x_{L}$ distributions photoproduction over DIS in Fig. 2 also shows the predictions of two models of absorption [3]. The model of D' Alesio and Pirner describes the shape of the data, with increasing absorption at decreasing values of $x_{L}$, but it predicts a lower value of the ratio than the data. $\gamma p$ interactions have a power law dependence on the photon-proton center-of-mass energy, $\sigma \propto W^{\lambda}$, with different values of $\lambda$ for DIS and photoproduction. Assuming that $\gamma \pi$ interactions have the same dependence, and recalling that $W_{\gamma \pi}=\sqrt{1-x_{L}} W_{\gamma p}$, the ratio of photoproduction and DIS cross sections is proportional to $\left(1-x_{L}\right)^{\Delta \lambda}$. After correcting the absorption factor of D' Alesio and Pirner for this differing $W$ dependence the model give a good description of the data.

Figure 5 shows the slopes $b$ for DIS, and the difference between slopes in photoproduction and DIS, $\Delta b$. Also shown are the predictions of a model of pion exchange incorporating neutron absorption and migration [8], and a model including in addition exchanges of $\rho$ and $a_{2}$ mesons [9]. The model with only pion exchange predicts too high a value of the slopes, but does predicts the correct magni-

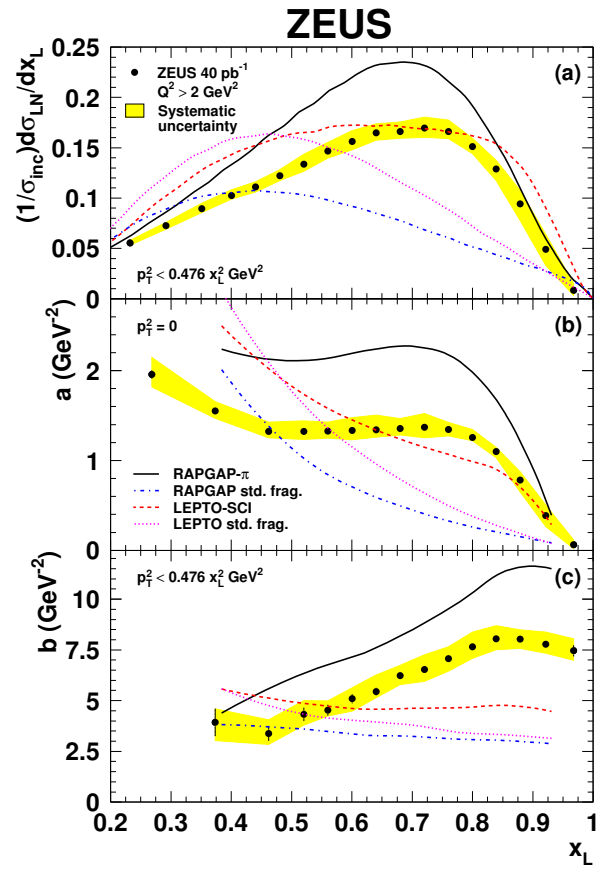

Figure 4: Leading neutron $x_{L}$ distributions and intercepts and slope for DIS, compared to Monte Carlo models.

tude of $\Delta b$. The model including also $\rho$ and $a_{2}$ exchanges gives a good description of the slopes, including the turnover at highest $x_{L}$. It also gives a fair prediction of the value of $\Delta b$. 


\section{Summary}

Leading neutron energy and $p_{T}$ distributions were measured in DIS and photoproduction. A decrease in neutron yield from high $Q^{2}$ DIS to very low $Q^{2}$ is indicative of absorption. Monte Carlo models incorporating only fragmentation of the proton remnant fail to describe the data. A pion exchange model gives a fair description of the energy distribution of neutron in DIS, but predicts too steep $p_{T}^{2}$ distributions. Addition of neutron absorption to the model describes the suppression seen in photoproduction. Adding further exchanges of $\rho$ and $a_{2}$ mesons gives a good description also of the $p_{T}^{2}$ distributions.

\section{References}

[1] ZEUS Coll., S. Chekanov et al., Nucl. Phys. B776 1 (2007), and references therein.

[2] M. Bishari, Phys. Lett. B38 510 (1972).

[3] N.N. Nikolaev, J. Speth and B.G. Zakharov, Preprint KFA-IKP(TH)-1997-17 (hepph/9708290), KFA-IKP (1997);

U. D'Alesio and H.J. Pirner, Eur. Phys. J. A7 109 (2000).

[4] G. Ingelman, A. Edin and J. Rathsman, Comp. Phys. Comm. 101108 (1997).

[5] H. Jung, Comp. Phys. Comm. 86147 (1995).

[6] T. Sjöstrand and M. Bengtsson, Comp. Phys. Comm. 43367 (1987).

[7] A. Edin, G. Ingelman and J. Rathsman, Phys. Lett. B366 371 (1996).

[8] A.B. Kaidalov et al., Eur. Phys. J. C47 385 (2006).

[9] V.A. Khoze, A.D. Martin and M.G. Ryskin, Preprint IPPP-06-36, DCPT-06-72 (hep$\mathrm{ph} / 0606213) 2006$.

[10] Slides:

http: //indico. cern. ch/contributionDisplay py? contribId=243\&sessionId=7\& conf Id=9499

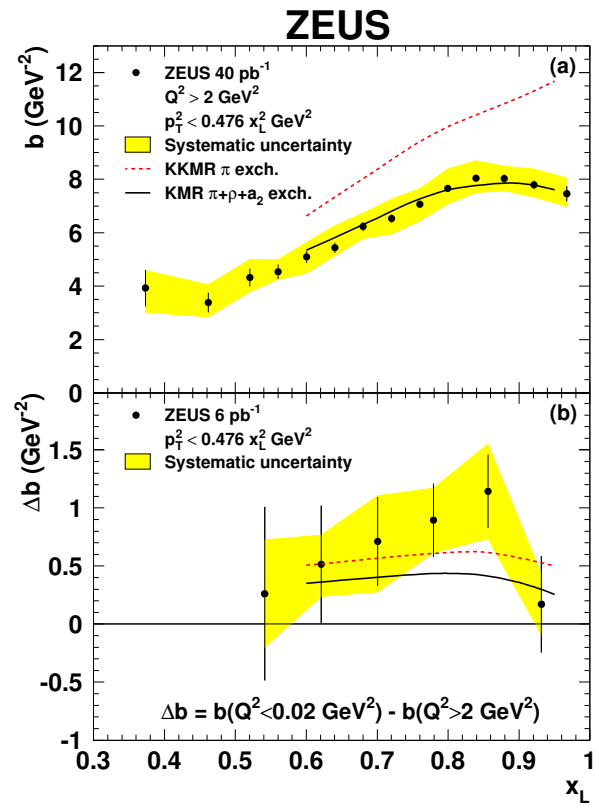

Figure 5: Neutron $p_{T}^{2}$ slopes in DIS (top) and difference in slopes between photoproduction and DIS (bottom). The dashed curve is a model of pion exchange including absorption; the solid curve is the model with the addition of $\rho$ and $a_{2}$ meson exchange. 